\title{
Executive and Non-Executive Functions in Attention Deficit Hyperactivity Disorder of the Inattentive Type (ADHD-I): A Cognitive Profile
}

\author{
Yongning Song ${ }^{1,2^{*}}$, Yuji Hakoda ${ }^{2}$ \\ ${ }^{1}$ Key Laboratory of Brain Functional Genomics (MOE \& STCSM), School of Psychology and Cognitive Science, \\ East China Normal University, Shanghai, China \\ ${ }^{2}$ Faculty of Human-Environment Studies, Kyushu University, Fukuoka, Japan \\ Email: *ynsong@psy.ecnu.edu.cn
}

Received November 25, 2013; revised December 29, 2013; accepted January 8, 2014

Copyright (C) 2014 Yongning Song, Yuji Hakoda. This is an open access article distributed under the Creative Commons Attribution License, which permits unrestricted use, distribution, and reproduction in any medium, provided the original work is properly cited. In accordance of the Creative Commons Attribution License all Copyrights (C) 2014 are reserved for SCIRP and the owner of the intellectual property Yong ning Song, Yuji Hakoda. All Copyright (c) 2014 are guarded by law and by SCIRP as a guardian.

\section{ABSTRACT}

Objective: This study examined the hypothesis that individuals with attention deficit hyperactivity disorder, predominantly inattentive type (ADHD-I), show both executive function (EF) deficits and non-EF deficits. Method: A group with ADHD-I $(n=16)$ and a paired control group $(n=21)$ completed a battery of tasks covering the major domains of EF (planning, working memory, flexibility and inhibition) and non-EF (alertness, divided attention, flexibility, sustained attention, visual field and visual scanning). Results: EF impairments in planning, spatial working memory, flexibility, and inhibition as well as non-EF impairments in divided attention, flexibility, sustained attention and visual scanning were observed in the ADHD-I group. Conclusion: Our results do not support the traditional model of ADHD. Given that neither EF nor non-EF tests typically distinguish ADHD-I from ADHD-C, alternative methodologies are required to confirm the validity of ADHD subtypes in the Diagnostic and Statistical Manual of Mental Disorders, Fourth Edition.

\section{KEYWORDS}

ADHD-I; Executive Function; Non-Executive Function; Cognitive Profile

\section{Introduction}

Executive function (EF) is an umbrella term in psychology that refers to the processes that control other cognitive processes [1]. Researchers have identified four distinct domains of EF: planning, working memory, flexibility, and response inhibition [2-6]. The role of EF is debated, but most researchers agree that EF is involved in deliberately managing an appropriate problem solving set to attain a future goal $[7,8]$.

A deficit in EF is postulated to account for core symptoms in psychiatric patients with no focal frontal lesions, such as those diagnosed with attention deficit hyperactivity disorder (ADHD). The evidence supporting a deficiency in EF domains in ADHD comes from a number of sources [8-14].

Clarification of the neuropsychological similarities and

"Corresponding author. differences in ADHD subtypes can contribute to understanding their etiological relationship. The Diagnostic and Statistical Manual of Mental Disorders [15] categorizes the symptoms of ADHD into three subtypes: ADHD, predominantly inattentive type (ADHD-I); ADHD, predominantly hyperactive-impulsive type (HD or ADHD$\mathrm{H}$ ); and ADHD, combined type (ADHD-C). In addition, Barkley postulated a model of ADHD in which only ADHD-C and ADHD-H, but not ADHD-I, were associated with EF deficits [16], yet it remained unclear whether the DSM-IV inattentive subtype involved distinct deficits [17].

Now, nearly all of the neuropsychological literature on ADHD pertains to the group designated as ADHD combined type (ADHD-C), while the primarily inattentive subtype of ADHD (ADHD-I) remains relatively underinvestigated with regard to potentially relevant cognitive functions [18-20]. Nigg suggested that further studies of 
children with ADHD-C versus controlling children on many executive measures might no longer be needed. Instead, studies to examine issues such as neuropsychological process theories of ADHD-I have been proposed [19].

Some research suggests that EF weaknesses are primarily associated with inattention [21-23]. If this is the case, we can further hypothesize that EF deficits are not specifically related to ADHD-C and ADHD-H. It is possible that those with ADHD-I suffer from the same neuropsychological impairment. Furthermore, combining the hypothesis that EF weaknesses are neither necessary nor sufficient to cause all cases of ADHD [8], we predict that individuals with ADHD-I not only encounter just difficulties with EF, but also show deficits in other cognitive domains (hereafter termed non-EF).

Thus, the first goal of the present study was to examine the EF weaknesses hypothesis in ADHD-I by comparing children with ADHD-I versus typically developing children in the four distinct EF domains of planing, working memory, flexibility, and inhibition. The second goal was to examine the non-EF deficit hypothesis in children with ADHD-I by comparing them with a control group on six non-EF domains: alertness, divided attention, flexibility, sustained attention, visual field and visual scanning.

\section{Methods}

\subsection{Participants}

The ADHD participants were recruited from several child psychiatry outpatient services across the Zabei istrict of Shanghai in China. Each sample was referred $f$ rom the Shanghai Pediatric Hospital where the participants were diagnosed with ADHD, primarily inattentive type, and engaged in twice-a-week attention training for special children at the support and rehabilitation center of the Zabei District of Shanghai.

Before testing, we confirmed that the children had not been on medication for at least 3 months. Before testing, parents were invited by letter to participate and provided their written informed consent. Each family was invited to participate in an unstructured screening interview based on the Child and Adolescent Psychiatric Assessment [24] at the support and rehabilitation center. Children's general information including medical history, developmental history, performance in family, and general symptoms were recorded. On the basis of the interview results, any child who had any other possible comorbid diagnosis, such as a significant learning disability, autistic spectrum disorder, or significant medical condition such as epilepsy, was excluded from further study.

Furthermore, a short version of Conners' Teacher Rating Scale (CTRS-S) [25] was completed for each child to confirm the pervasiveness of symptoms. The CTRS-S contains 27 items comprising four subscales: oppositional, cognitive problems/inattention, hyperactivity, and ADHD index. Scoring was performed according to the test manual [26], with each raw score converted to a $t$ score dependent on age and sex of the child. Established cutoff points for possible and likely ADHD, primarily inattentive type [26], were imposed, and any child who did not meet CTRS-S criteria was dropped from further study. In addition, all children met DSM-IV (APA) [27] diagnostic criteria for ADHD. Any child with ADHD-H or ADHD-C was excluded. Considering that level of intelligence may affect performance on EF tests, all children were first administered an IQ test (Combined Raven's Test, CRT), and children with mental retardation (IQ scores below 75) were excluded. Finally, a total of 16 children with ADHD-I participated in the experiment. Twenty-one children without ADHD were paired with the ADHD group by gender and age. A teacher completed the CTRS-S [25] for the control group, with t scores below 50 used to confirm the children's nonADHD status. In addition, the non-ADHD group was administered the IQ test (CRT) to confirm that they did not have mental retardation. Information on participants is shown in Table 1.

\subsection{EF Tests}

We chose EF tests according to distinct domains of EF: planning, working memory, flexibility, and response inhibition [2-6]. Four EF tests were conducted in this study: the Spatial Working Memory Test (SWM), Stockings of Cambridge Test (SOC), Wisconsin Card Sorting Test (WCST), and Stroop/reverse-Stroop Test. With regard to response inhibition, Barkley proposed a model suggesting that a deficit in behavioral inhibition, considered a key process in EF, accounts for central impairment of ADHD [16]. In the model, Barkley distinguished three interrelated processes believed to constitute behavioral inhibition: 1) inhibition of a prepotent response; 2) cessation of anongoing response; and 3) interference control. Researchers have long used Stroop/reverse-Stroop interference as the main paradigm to study interference control. Thus, in this study, we used the Stroop/reverse-

Table 1. Group means (SDs) for age and IQ.

\begin{tabular}{cccc}
\hline & ADHD-I & Control & P value \\
\hline $\mathrm{N}$ & 16 & 21 & \\
Sex ratio (F/M) & $12 / 4$ & $14 / 7$ & Ns \\
Age & $12(1.43)$ & $12(1.41)$ & Ns \\
Range of age & $9-14$ & $9-14$ & \\
IQ(CRT-C) & $89(11.3)$ & $92(12)$ & $p=0.51$ \\
Range of IQ & $75-122$ & $80-123$ & \\
\hline
\end{tabular}


Stroop Test $[28,29]$ to evaluate the level of response inhibition.

Spatial Working Memory Test (altered). This test was designed based on the SWM in the Cambridge Automated Neuropsychological Test Battery (CANTAB) [30,31]. In this test, participants are asked to search through a number of boxes presented on the screen to find a token. The key instruction states that once a token has been found in a box, that box cannot be used again to hide a token during that particular trial. On each trial, the total number of blue tokens to be found corresponds to the number of boxes on the screen. Once a blue token is found in a particular box, that box cannot be used again to hide a token. Returning to an empty box where a target has already been found is referred to as a "betweensearch error".

In the original SWM, there were four types of trials with either three, four, six, or eight boxes in each. Several previous studies showed that children with ADHD made significantly more errors compared with controls only on the eight-box problems [32,33]. Given that between-search errors may appear as a function of the number of boxes in pediatric clinical populations [34], it is possible that children with ADHD also make significantly more errors compared with controls on seven-box problems. Thus, we made a few changes to the original task, in which the independent variable of box consisted of five uninterrupted levels with three, four, five, six, or seven boxes. There were four test trials each with three, four, five, six, and seven boxes. The order of the trials was randomized, with the constraint that the same number of boxes did not occur consecutively. The dependent measure for the SWM test was between-search errors on three-, four-, five-, six-, and seven-box problems.

Stocking of Cambridge Test (altered). This test was designed based on the SOC in the CANTAB [30,31]. This test is closely related to the Tower of London task developed by Shallice and McCarthy [35]. In this test, two sets of three colored balls (one green, one blue, and one red) were presented on the screen. Participants were asked to rearrange the balls in the bottom display such that their positions matched the "goal" arrangement in the tophalf of the screen. The starting position of the balls was varied so that in any particular trial the solution could only be reached after a minimum of two, three, four, or five moves. Participants were instructed to examine the position of the balls at the beginning of each problem and attempt to solve it in the minimum possible number of moves. The time to complete the pattern is taken as a measure of the participant's planning ability.

In the original SOC task, there were four test trials each with two, three, four, and five moves. However, some studies failed to find that children with ADHD made significantly more extra moves than typically de- veloping children on this task $[32,33,36]$. It is possible that the short range of the minimum moves to goal state can account for the above conclusion. Thus, we made a few changes to the original task, such that the minimum moves to goal state ranged from three to seven moves. There were four test trials each with of three, four, five, six, and seven moves, and the order of the trials was randomized. The dependent measure for the SWM test was thinking time on three-, four-, five-, six-, and seven-move problems.

Wisconsin Card Sorting Test. Flexibility was assessed with the Computerized WCST [37-39], a widely used test to measure cognitive flexibility or set shifting. In this test, participants were asked to match a series of stimulus cards to a set of four target cards that differed by form, color, and number. The display remained until a choice was made. Feedback followed the response, and consisted of a " $\times$ " sign if the response was correct, or a ${ }^{\text {c" }}$ " sign if the response was incorrect. Response cards could be matched by color (red, green, blue, yellow), shape (triangle, star, cross, circle), or number $(1,2,3,4)$. After participants determined one of the correct dimensions, referred to as "categories," 10 correct responses were required before the category was shifted to the next one. The task was terminated after a maximum of 128 trials was reached. The order of the sorting principles was randomized, with the constraint that the same sorting principles did not occur consecutively.

Continued matching to a category that is no longer correct is considered a perseverative error. Other errors that occur when a participant is required to switch to another sorting principle are referred to as non-perseverative errors. The variables of interest were the number of categories achieved, percentage of perseverative errors and percentage of non-perseverative errors.

The Stroop/reverse-Stroop Test. The Stroop/reverseStroop Test $[28,29,40]$ was used to evaluate thelevel of both Stroop interference and reverse-Stroop interference. The test comprised four subtests in which all color-word combinations and color patches were printed on four separate sheets of paper. Test 1 was the reverse-Stroop interference control condition, in which all the colorword combinations were written in black ink, requiring participants to make a choice from five matching colors printed on the right side of the test sheet corresponding to the color-word patches on the left side of the test sheet. Test 2 was the reverse-Stroop interference condition, in which incongruent color-word combinations were shown on the left side of the test sheet, requiring participants to make a choice from five matching color patches on the right side of the test sheet corresponding to the semantic meaning of the word on the left side of the test sheet. Test 3 was the Stroop interference control condition in which the color patch was shown on the left side of the 
test sheet, requiring participants to make a choice from five matching color-word patches (written in black ink) on the right side of the test sheet corresponding to the color of the color patch on the left side ofthe test sheet. Test 4 was the Stroop interference condition, in which incongruent color-word cards were shown on the left side of the test sheet, requiring participants to make achoice from five matching color-word patches (printed with black ink) according to the ink color of the color-word combination on the left side of the test sheet.

Each test consisted of 10 practice trials and 100 test trials. On the basis of the number of correct responses in each subtest (C1, C2, C3, C4), two interference ratios were calculated using the following formulas: Stroop interference ratio, $(\mathrm{SI})=(\mathrm{C} 3-\mathrm{C} 4) / \mathrm{C} 3$, and reverse-Stroop interference ratio, $(\mathrm{RI})=(\mathrm{C} 1-\mathrm{C} 2) / \mathrm{C} 1$.

\subsection{Non-EF Tests}

Previous studies have shown that individuals with ADHD-I have a deficit in focused or selective attention [41,42], while the deficit in ADHD-C has been characterized as sustained persistence and distractibility [16]. To fully assess non-EF in this study, performance was assessed by a set of computer-assisted psychological tests, the Test for Attentional Performance (TAP), version 2.2, published by Zimmermann and Fimm [43]. The six subtests of alertness, divided attention, flexibility, sustained attention, visual field, and visual scanning were administered.

Alertness Test (TAP, subtest 1). A simple reaction time (RT) task measures response readiness to a simple visual target on the computer screen. Simple reaction time has been shown to be a valid measure of general slowness. In this test, a cross $(2 \mathrm{~cm})$ appeared in the middle of the screen, and the participant had to press a button as rapidly as possible. The interval between the warning and the imperative stimulus varied randomly between 300 and $700 \mathrm{~ms}$. The reaction times (RTs) were automatically recorded by the program (a total of 40 trials were presented in this subtest).

Divided Attention Test (TAP, subtest 5). In this subtest, participants had to deal with one visual simultaneous task. The visual task consisted of a matrix of $4 \times 4$ dots (size: $10 \times 10 \mathrm{~cm}$ ). Seven small Xs were superimposed randomly over the $4 \times 4$ dots. When four Xs formed a square, the participants had to react as quickly as possible by pressing a button. The task contained 20 visual targets out of 20 visual non-targets.

Flexibility Test (TAP, subtest 6). In this subtest, one letter and one digit were presented simultaneously, one on the left and one on the right. The digit always represented the targets timulus (50 trials). Participants needed to respond to each trial by pressing the corresponding left or right response button as quickly and as accurately as possible to judge which side of the target was displayed. The placement of the target was randomized so that participants could not anticipate where it would be displayed.

Sustained Attention Test (TAP, subtest 9). In this subtest, a sequence of stimuli was presented on the monitor. The stimuli varied in a range of feature dimensions: color, shape, size and filling. A target stimulus occurred whenever it corresponded in one predetermined stimulus dimension with the preceding stimulus (e.g., the same shape but with different color, size and filling), participants needed to respond to each trial by pressing the space key as quickly as possible (test time lasted $10 \mathrm{mi}-$ nutes).

Visual FieldTest (TAP, subtest 11). To record vision in circumscribed areas of the visual field, a stimulus was presented at different points of the screen and at varying intervals. Participants were required to fixate on the middle of the screen throughout the entire test run. Whenever the peripheral stimulus appeared the patient was to press the reaction key as quickly as possible. The RTs to targets were recorded automatically. A total of 40 stimuli were presented in this subtask.

Visual scanning Test (TAP, subtest 12). In this subtest, a matrix-like arrangement of $5 \times 5$ stimuli was used. Participants were required to detect whether this arrangement included a critical stimulus. One reaction key was used for the answer "present" and another for the answer "not present". The task contained 20 visual targets out of 20 visual non-targets.

\subsection{Procedure}

Testing took place on four different occasions and was administered in a fixed order for both groups. During the first session, the Stroop/reverse-Stroop Test was administered. In the second testing session, the TAP battery was administered individually. In the third testing session, the WCST and SOC were administered individually. Finally, the SWM was administered individually.

\section{Results}

In this section, we briefly provide the statistical analyses, focusing on the performance on the EF tests (working memory, planning, flexibility and inhibition) and nonEF tests (alertness, divided attention, flexibility, sustained attention, visual field and visual scanning) between children with ADHD-I and typically developing children.

\subsection{EF Tests}

The means and standard deviations (SD) on EF tests are shown in Table 2.

Working memory. We performed a two-way analysis 
Table 2. Means and standard deviations (SD) on EF tests.

\begin{tabular}{|c|c|c|c|c|}
\hline EF domain & Tasks & ADHD-I & Control & Contrast \\
\hline \multirow[t]{6}{*}{ Working Memory } & SWM & & & \\
\hline & BSE on 3-box problems & $0.13(.34)$ & $0.01(.22)$ & Ns \\
\hline & BSE on 4-box problems & $1.31(1.85)$ & $0.43(1.03)$ & Ns \\
\hline & BSE on 5-box problems & $0.88(1.41)$ & $0.71(1.82)$ & Ns \\
\hline & BSE on 6-box problems & $4.38(4.56)$ & $1.81(2.52)$ & ADHD-I > NC \\
\hline & BSE on 7-box problems & $9.83(7.95)$ & $2.79(4.15)$ & ADHD-I > NC \\
\hline \multirow[t]{6}{*}{ Planning } & SOC & & & \\
\hline & TT on 3-move problems & $17.27(6.71)$ & $14.20(3.41)$ & Ns \\
\hline & TT on 4-move problems & 27.76(15.18) & 33.14(11.80) & Ns \\
\hline & TT on 5-move problems & 62.81(38.92) & $37.05(31.08)$ & ADHD-I > NC \\
\hline & TT on 6-move problems & $70.68(30.00)$ & 49.24(20.64) & ADHD-I > NC \\
\hline & TT on 7-move problems & $73.20(39.23)$ & 48.32(37.85) & ADHD-I > NC \\
\hline \multirow[t]{4}{*}{ Flexibility } & WCST & & & \\
\hline & Categories & $6.50(2.00)$ & $8.29(1.55)$ & ADHD-I $<\mathrm{NC}$ \\
\hline & Perseverative errors & $13.94(9.47)$ & $6.76(8.31)$ & ADHD-I > NC \\
\hline & Non-perseverative errors & $25.44(5.97)$ & 23.10(5.88) & Ns \\
\hline \multirow[t]{3}{*}{ Inhibition } & Stroop/reverse-Stroop Test & & & \\
\hline & SI & $0.23(.21)$ & $0.19(.13)$ & Ns \\
\hline & RI & $0.30(.15)$ & $0.17(.11)$ & ADHD-I > NC \\
\hline
\end{tabular}

Note: ADHD-I = ADHD Pre-dominantly Inattentive type; SWM: Spatial Working Memory ; SOC = Stocking of Cambridge; WCST = Wisconsin Card Sorting Test; TT: Thinking Times; BSE = Between Search Errors; SI = Stroop Interference ratio; RI reverse-Stroop Interference ratio.

of variance (ANOVA) with Planning. We performed a two-way ANOVA with the group type (ADHD-I or difficulty (three- to seven-box problems) as a within participants factor. Results showed that the two main effects of group type, $F(1,35)=10.37, p<0.01$, and task difficulty, $F(4,140)=27.54, p<0.01$, were significant. The interaction effect between group type and task difficulty was significant, $F(4,140)=9.01, p<0.01$. Furthermore, the simple main effect of group type was only significant in six-box problems, $F(1,35)=4.77, p=0.04$ and seven-box problems, $F(1,35)=12.18, p<0.01$. It was not significant in three-box problems, $F(1,35)=0.70, p=$ 0.41 , four-box problems, $F(1,35)=3.42, p=0.07$, or five-box problems, $F(1,35)=.01, p=0.77$.

Planning. We performed a two-way ANOVA with the group type (ADHD-I or control) as a between- participants factor and the task difficulty (three- to seven-move problems) as a within participants factor. Results showed that the two main effects of group type, $F(1,35)=10.17$, $p<0.01$, and task difficulty, $F(4,140)=21.32, p<0.01$, were significant. The interaction effect between group type and task difficulty was significant, $F(4,140)=2.77$, $p<0.05$. Furthermore, the simple main effect of group type was only significant in five-move problems, $F(1,35)$ $=5.02, p<0.05$, six-move problems, $F(1,35)=6.63, p<$ 0.05 , and seven-move problems, $F(1,35)=3.80, p<$ 0.05 . It was not significant in three-move problems, $F(1$, $35)=3.29, p=0.08$, and four-move problems, $F(1,35)$ $=0.48, p=0.23$.

Flexibility. We performed an independent-samples $\mathrm{t}$ test on the categories achieved by the two groups. Re- sults showed that the effect of group type, $t(35)=3.06, p$ $<0.01$, was significant. In addition, we performed a two-way ANOVA with the group type as a betweenparticipants factor and the error type (perseverative errors and non-perseverative errors) as a within participants factor. Results showed that the two main effects of group type, $F(1,35)=4.20, p<0.05$, and error type, $F(4,140)$ $=233.91, p<0.01$, were significant. The interaction effect between group type and test condition was significant, $F(4,140)=7.05, p<0.01$. Furthermore, the simple main effect of group type was only significant in perseverative errors, $F(1,35)=6.01, p<0.05$. It was not significant in non-perseverative errors, $F(1,35)=1.42, p=$ 0.24 .

Inhibition. We performed a two-way ANOVA with the group type (ADHD-I or control) as a between-participants factor and the interference type (SI and RI) as a within participants factor. Results showed that the two main effects of group type, $F(1,35)=3.63, p=0.06$, and interference type, $F(1,35)=0.76, p=0.39$, were not significant. The interaction effect between group type and interference type was significant, $F(1,35)=3.52, p$ $<0.05$. Furthermore, the simple main effect of group type was only significant in reverse-Stroop interference, $F(1,35)=7.52, p<0.01$, whereas Stroop interference was not significant, $F(1,35)=.37, p=0.55$.

\subsection{Non-EF Tests}

The means and standard deviations (SD) on Non-EF tests are shown in Table 3. 
Table 3. Means and standard deviations (SD) on non-EF tests.

\begin{tabular}{ccccc}
\hline & Tasks & ADHD-I & Control & Contrast \\
\hline Alertness & TAP, subtest 1 & $350.67(54.44)$ & $345.17(64.88)$ & Ns \\
Divided attention & TAP, subtest 4 & $1434.17(432.73)$ & $1097.09(265.93)$ & ADHD-I $<$ NC \\
Flexibility & TAP, subtest 6 & $560.75(92.43)$ & $480.00(74.42)$ & ADHD-I $<$ NC \\
Sustained attention & TAP, subtest 9 & $560.56(59.50)$ & $515.96(61.07)$ & ADHD-I $<$ NC \\
Visual field & TAP, subtest 11 & $503.97(107.96)$ & $496.36(90.83)$ & Ns \\
Visual scanning & TAP, subtest 12 & $5454.91(1270.07)$ & $4114.94(898.77)$ & ADHD-I $>$ NC \\
\hline
\end{tabular}

Note: TAP $=$ Test for Attentional Performance.

We performed multivariate analysis (Pillai's trace) with group type (ADHD group or non-ADHD group) as a fixed factor and RTs on the sixsubtests as a dependent factor. Results showed that the effect of group type, $F(6$, $28)=4.42, p<0.01$, was significant. The tests of between-subjects effect indicated that the group type effect was significant for divided attention, $F(1,35)=8.18, p<$ 0.01 , flexibility, $F(1,35)=8.15, p<0.01$, sustained attention, $F(1,35)=4.57, p<0.05$, and visual scanning, $F(1,35)=13.41, p<0.01$. It was not significant for alertness, $F(1,35)=0.07, p=0.98$, or visual field, $F(1$, 35) $=0.06, p=0.79$.

\section{Discussion}

\subsection{EF Domains}

Working memory. Results of the two-way ANOVA indicated a significant group-by-task difficulty interaction: the ADHD-I differed significantly from controls only on six- and seven-box problems. In contrast to three- and four-box problems, in six- and seven-box problems there is a higher memory load of the task. Our findings indicate that the higher memory load task affected the ADHD-I group more than the controls. This result is consistent with several previous studies in which children with ADHD-C exhibited deficits in multiple components of working memory $[44,32,45]$. These results suggest that working memory is impaired in both ADHD-C and ADHD-I.

Planning. Analysis of thinking times on three-, four-, five-, six- and seven-move problems indicated that children with ADHD-I took more time to complete the five-, six- and seven-move problems than controls. The current findings are in contrast to previous studies [46, 47] that failed to find a significant divergence between ADHD-C and controls on three difficulty levels of SOC: two or three moves necessary to solve the problem (lowest difficulty level), four moves (medium difficulty level), and five moves (highest difficulty level). We argued that the short level range (the maximum move to goal state is five) may account for the no-difference findings in these studies. Numerous studies $[8,33]$ have reported poor performance on planning tasks in individuals with ADHD-C.
Evidence from our research and previous studies support the hypothesis that planning is impaired in both ADHD$\mathrm{C}$ and ADHD-I.

Flexibility. Results showed that the categories achieved by the ADHD group were fewer than the categories achieved by controls, and the simple main effect of group type was only significant for perseverative errors and not significant for non-perseverative errors. This indicates that the ADHD-I group exhibited a deficit in flexibility relative to typically developing children. Although a few studies have shown no statistically significant differences from controls on the WCST in individuals with ADHD $[46,48,49]$, more than half of the investigations have shown statistically significant differences from controls [14,50-53]. Moreover, Houghton et al. found no differences between inattentive and combined subtypes on WCST $[23,54]$. These results support the hypothesis that flexibility is impaired in both ADHD-C and ADHD-I.

Inhibition. We found an asymmetric phenomenon between Stroop interference and reverse-Stroop interference for ADHD-I participants. This finding replicated our recent results reporting an ADHD-I impairment in reverse-Stroop interference but not in Stroop interference [40]. A number of studies have used the Stroop test to examine interference control in ADHD, but results have been inconsistent. Recently, Mourik, Oosterlaan, Sergeant completed a meta-analytic review that systematically examined 17 studies of Stroop interference control in ADHD [55]. They concluded that the Stroop colorword task does not provide strong evidence for a deficit in interference control in ADHD. Lansbergen, Kenemans, and Van Engeland conducted another meta-analytic review of 19 studies that administered the Stroop tests to groups with ADHD [56]. In contrast, consistency analysis of ratio scores across those 19 studies revealed that interference control was consistently compromised in individuals with ADHD. We have few studies available on reverse-Stroop interference in ADHD-C. This is possibly because the reverse-Stroop has seldom been discussed in ADHD because it cannot be observed in oral responses [57]. It is difficult to conclude that individuals with ADHD-I or ADHD-C exhibit the same deficit in reverse-Stroop interference until more studies on this 
question are conducted.

\subsection{Non-EF Domains}

Deficits in divided attention, flexibility, sustained attention, and visual scanning relative to controls indicate that individuals with ADHD-I also exhibited impairment on the non-EF domains. Willcutt et al. suggested that EF weaknesses are neither necessary nor sufficient to cause all cases of ADHD [8]. Our research confirms that ADHD-I is associated with deficits in EF as well as non-EF domains. The DSM-IV does not provide specific examples of the cognitive difference between ADHD-C and ADHD-I. With regard to non-EF domains, previous studies have suggested that ADHD-I shows a deficit in speed of information processing, generally, and in focused or selective attention, specifically [41,42], while deficits in ADHD-C are characterized as sustained persistence. In the present research, we found that ADHD-I is also associated with a sustained attention deficit [16]. Moreover, Geurts, Vert'ec, Oosterlaana, Roeyersc, and Sergeanta found no differences between inattentive and combined ADHD subtypes on non-EF tasks, such as response execution, short-term memory, visual-motor integration and categorization [47]. Based on combined results of the current research and previous studies, we posit that deficits in non-EF cognitive ability in ADHD-I may be the same as those found in ADHD-C.

\section{General Discussion}

The present study was designed to investigate the hypothesis that those with ADHD-I exhibit both EF deficits and non-EF deficits by comparing typically developing controls with boys carefully diagnosed with ADHD-I on an extensive battery of tasks that cover the major EF and non-EF domains.

With regard to the EF domains, results are consistent with findings in previous studies of EF and ADHD. That is, ADHD is associated with weaknesses in several key EF domains, but the strongest and most consistent effects are obtained on measures of response inhibition, vigilance, spatial working memory and some measures of planning $[8,22,46,47,58]$. The deficits on EF domains revealed in ADHD-I also suggest that the pathology of ADHD-I is related to deficits in managing an appropriate problem or attaining a future goal. Furthermore, results did not yield evidence for the model of ADHD in which only ADHD-C and ADHD-H, but not ADHD-I, are associated with EF deficits [16].

With regard to non-EF domains, findings revealed that the children with ADHD-I also demonstrated deficits in these domains, such as divided attention, flexibility, sustained attention and visual scanning. This suggests that children with ADHD-I not only show deficits in EF, but also experience deficits in other non-EF domains.

Discriminating among disorders is particularly important. However, there are no objective diagnostic tests for ADHD-I [59]. Considering the fact that neither EF nor non-EF domains distinguish ADHD-I from ADHD-C, examination of other factors, such as social, emotional and behavioral characteristics [60,61] may be needed to support the validity of ADHD subtypes in the DSM-IV.

\section{Limitations}

A limitation of our study findings is the small sample size and potential response bias from those who agreed to participate. To gather more reliable data and validate the results of the present study, future research should focus on selecting larger samples to engage in the same tasks. Furthermore, to examine whether EF and non-EF tests can distinguish ADHD-I from ADHD-C, it would be useful to make a direct comparison between ADHD-C and ADHD-I in the battery of EF and non-EF tests used in the study. Future studies should be conducted by using the same tasks with an ADHD-C group.

\section{Acknowledgements}

The authors wish to thank all the participants in this experiment. Special thanks go to Yu Jia for her invaluable help. This work was supported by National Natural Foundation of China (31300839). This work was also sponsored by the Japan Society for the Promotion of Science (JSPS) Postdoctoral Fellowship for Foreign Researchers KAKENHI (25-03311), Shanghai Pujiang Program (12PJC034), MOE (Ministry of Education in China) Project of Humanities and Social Sciences (13YJC190020) and SRF for ROCS, SEM. We declare that there is no conflict of interest in this paper.

\section{REFERENCES}

[1] R. C. K. Chan, D. Shum, T. Toulopoulou, et al., "Assessment of Executive Functions: Review of Instruments and Identification of Critical Issues," Archives of Clinical Neuropsychology, Vol. 23, No. 2, 2008, pp. 201-216. http://dx.doi.org/10.1016/j.acn.2007.08.010

[2] C. Hughes, "Executive Function in Preschoolers: Links with Theory of Mind and Verbal Ability,” British Journal of Developmental Psychology, Vol. 16, No. 2, 1998, pp. 233-253.

http://dx.doi.org/10.1111/j.2044-835X.1998.tb00921.x

[3] A. Miyake, N. P. Friedman, M. J. Emerson, et al., "The Unity and Diversity of Executive Functions and Their Contributions to Complex Frontal Lobe Tasks: A Latent Variable Analysis,” Cognitive psychology, Vol. 41, No. 1, 2000, pp. 49-100.

http://dx.doi.org/10.1006/cogp.1999.0734

[4] S. Ozonoff, "Components of Executive Function in Autism and Other Disorders,” In: J. Russell, Ed., Autism as 
an Executive Disorder, Oxford University Press, New York, 1997.

[5] T. W. Robbins, D. Weinberger, J. G. Taylor, et al., "Dissociating Executive Functions of the Prefrontal Cortex," Philosophical Transactions of the Royal Society of London. Series B: Biological Sciences, Vol. 351, No. 1346, 1996, pp. 1463-1471. http://dx.doi.org/10.1098/rstb.1996.0131

[6] M. C. Welsh, B. F. Pennington and D. B. Groisser, “A Normative-Developmental Study of Executive Function: A Window on Prefrontal Function in Children,” Developmental Neuropsychology, Vol. 7, No. 2, 1991, pp. 131149. http://dx.doi.org/10.1080/87565649109540483

[7] M. C. Welsh and B. F. Pennington, "Assessing Frontal Lobe Functioning in Children: Views from Developmental Psychology,” Developmental Neuropsychology, Vol. 4, No. 3, 1988, pp. 199-230.

http://dx.doi.org/10.1080/87565648809540405

[8] E. G. Willcutt, A. E. Doyle, J. T. Nigg, et al., "Validity of the Executive Function Theory of Attention-Deficit/ Hyperactivity Disorder: A Meta-Analytic Review,” Biological Psychiatry, Vol. 57, No. 11, 2005, pp. 1336-1346. http://dx.doi.org/10.1016/j.biopsych.2005.02.006

[9] L. L. Boucugnani and R. W. Jones, "Behaviors Analogous to Frontal Lobe Dysfunction in Children with Attention Deficit Hyperactivity Disorder," Archives of Clinical Neuropsychology, Vol. 4, No. 2, 1989, pp. 161-173.

[10] F. X. Castellanos and R. Tannock, "Neuroscience of Attention-Deficit/Hyperactivity Disorder: The Search for Endophenotypes," Nature Reviews Neuroscience, Vol. 3, No. 8, 2002, pp. 617-628.

[11] P. M, Levin, "Restlessness in Children,” Archives of Neurology and Psychiatry, Vol. 39, No. 4, 1938, pp. 764-770. http://dx.doi.org/10.1111/j.1469-7610.1996.tb01380.x

[12] B. F. Pennington and S. Ozonoff, "Executive Functions and Developmental Psychopathology,” Journal of Child Psychology and Psychiatry, Vol. 37, No. 1, 1996, pp. 5187. http://dx.doi.org/10.1111/j.1469-7610.1996.tb01380.x

[13] D. A. Pineda, M. A. Restrepo, R. J. Sarmiento, et al., "Statistical Analyses of Structural Magnetic Resonance Imaging of the Head of the Caudate Nucleus in Colombian Children with Attention-Deficit Hyperactivity Disorder,” Journal of Child Neurology, Vol. 17, No. 2, 2002, pp. 97-105. http://dx.doi.org/10.1177/088307380201700202

[14] K. L. Shue and V. I. Douglas, "Attention Deficit Hyperactivity Disorder and the Frontal Lobe Syndrome,” Brain and Cognition, Vol. 20, No. 1, 1992, pp. 104-124. http://dx.doi.org/10.1016/0278-2626(92)90064-S

[15] American Psychiatric Association (APA), "Diagnostic and Statistical Manual of Mental Disorders," 4th Edition, Text Revision, Author, Washington DC, 2000.

[16] R. A. Barkley, "Behavioral Inhibition, Sustained Attention, and Executive Functions: Constructing a Unifying Theory of ADHD," Psychological Bulletin, Vol. 121, No. 1, 1997, p. 65. http://dx.doi.org/10.1037/0033-2909.121.1.65

[17] C. L. Carlson and M. Mann, "Attention-Deficit/Hyperactivity Disorder, Predominately Inattentive Subtype,”
Child and Adolescent Psychiatric Clinics of North America, Vol. 19, No. 9, 2000, pp. 499-510.

[18] R. Milich, A. C. Balentine and D. R. Lynam, “ADHD Combined Type and ADHD Predominantly Inattentive Type Are Distinct and Unrelated Disorders," Clinical Psychology: Science and Practice, Vol. 8, No. 4, 2001, pp. 463-488. http://dx.doi.org/10.1093/clipsy.8.4.463

[19] J. T. Nigg, "Neuropsychologic Theory and Findings in Attention-Deficit/Hyperactivity Disorder: The State of the Field and Salient Challenges for the Coming Decade," Biological Psychiatry, Vol. 57, No. 11, 2005, pp. 14241435. http://dx.doi.org/10.1016/j.biopsych.2004.11.011

[20] J. T. Nigg, L. G. Blaskey, C. L. Huang-Pollock, et al., "Neuropsychological Executive Functions and DSM-IV ADHD Subtypes," Journal of the American Academy of Child \& Adolescent Psychiatry, Vol. 41, No. 1, 2002, pp. 59-66. http://dx.doi.org/10.1097/00004583-200201000-00012

[21] A. C. Bedard, A. Ickowicz, G. D. Logan, et al., "Selective Inhibition in Children with Attention-Deficit Hyperactivity Disorder off and on Stimulant Medication," Journal of Abnormal Child Psychology, Vol. 31, No. 3, 2003, pp. 315-327. http://dx.doi.org/10.1023/A:1023285614844

[22] N. Chhabildas, B. F and Pennington, E. G. Willcutt, “A Comparison of the Neuropsychological Profiles of the DSM-IV Subtypes of ADHD,” Journal of Abnormal Child Psychology, Vol. 29, No. 6, 2001, pp. 529-540. http://dx.doi.org/10.1023/A:1012281226028

[23] M. Schmitz, L. Cadore, M. Paczko, et al., "Neuropsychological Performance in DSM-IV ADHD Subtypes: An Exploratory Study with Untreated Adolescents," Canadian Journal of Psychiatry, Vol. 47, 2002, pp. 863-869.

[24] A. Angold, M. Prendergast, A. Cox, et al., "The Child and Adolescent Psychiatric Assessment (CAPA)," Psychological Medicine, Vol. 25, No. 4, 1995, pp. 739-754. http://dx.doi.org/10.1017/S003329170003498X

[25] C. K. Conners, "Rating Scales in Attention-Deficit/Hyperactivity Disorder: Use in Assessment and Treatment Monitoring,” The Journal of Clinical Psychiatry, Vol. 59, No. 9, 1997, pp. 24-30.

[26] C. K. Conners, "The Conners Rating Scales: Instruments for the Assessment of Childhood Psychopathology," Duke University, Durham, 1995.

[27] American Psychiatric Association, "Diagnostic and Statistical Manual of Mental Disorders: DSM-IV,” American Psychiatric Pub Inc., Arlington, 1994.

[28] Y. Hakoda and M Sasaki, "Group Version of the Stroop and Reverse-Stroop Test: The Effects of Reaction Mode, Order and Practice,” Kyoiku Shinrigaku Kenkyu (Japanese Journal of Educational Psychology), Vol. 38, No. 1, 1990, pp. 389-394.

[29] Y. Hakoda and M. Sasaki, "Two Interferences Based on New Stroop Test and Reaction Model (Report No. 3)," Kyushu University, Department of Information Systems, Fukuoka, 1991, pp. 12-15.

[30] M. Luciana and C. A. Nelson, "Neurodevelopmental Assessment of Cognitive Function Using the Cambridge Neuropsychological Testing Automated Battery (CAN- 
TAB): Validation and Future Goals,” Functional Neuroimaging in Child Psychiatry, Cambridge University Press, Cambridge, 2000, pp. 379-397.

[31] B. J. Sahakian and A. M. Owen, "Computerized Assessment in Neuropsychiatry Using CANTAB: Discussion Paper,” Journal of the Royal Society of Medicine, Vol. 85, No. 7, 1992, p. 399.

[32] M. C. Goldberg, S. H. Mostofsky, L. E. Cutting, et al., "Subtle Executive Impairment in Children with Autism and Children with ADHD," Journal of Autism and Developmental Disorders, Vol. 35, No. 3, 2005, pp. 279-293. http://dx.doi.org/10.1007/s10803-005-3291-4

[33] S. Kempton, A. Vance, P. Maruff, et al., "Executive Function and Attention Deficit Hyperactivity Disorder: Stimulant Medication and Better Executive Function Performance in Children,” Psychological Medicine, Vol. 29, No. 3, 1999, pp. 527-538. http://dx.doi.org/10.1017/S0033291799008338

[34] E. Joyce, S. Blumenthal and S. Wessely, "Memory, Attention, and Executive Function in Chronic Fatigue Syndrome,” Journal of Neurology, Neurosurgery \& Psychiatry, Vol. 60, No. 5, 1996, pp. 495-503. http://dx.doi.org/10.1136/jnnp.60.5.495

[35] T. Shallice, “Specific Impairments of Planning," Philosophical Transactions of the Royal Society of London, Biological Sciences, Vol. 298, No. 1089, 1982, pp. 199209. http://dx.doi.org/10.1098/rstb.1982.0082

[36] M. Luciana, "Practitioner Review: Computerized Assessment of Neuropsychological Function in Children: Clinical and Research Applications of the Cambridge Neuropsychological Testing Automated Battery (CANTAB)," Journal of Child Psychology and Psychiatry, Vol. 44, No. 5, 2003, pp. 649-663. http://dx.doi.org/10.1111/1469-7610.00152

[37] D. A. Grant and E. Berg, “A Behavioral Analysis of Degree of Reinforcement and Ease of Shifting to New Responses in a Weigl-Type Card-Sorting Problem,” Journal of Experimental Psychology, Vol. 38, No. 4, 1948, pp. 404-411. http://dx.doi.org/10.1037/h0059831

[38] R. K. Heaton, “A Manual for the Wisconsin Card Sorting Test,” Western Psycological Services, 1981.

[39] R. K. Heaton, "Wisconsin Card Sorting Test: Computer Version 2,” Psychological Assessment Resources, Odessa, 1993.

[40] Y. Song and Y. Hakoda, "An Asymmetric Stroop/ReverseStroop Interference Phenomenon in ADHD," Journal of Attention Disorders, Vol. 15, No. 6, 2011, pp. 499-505. http://dx.doi.org/10.1177/1087054710367607

[41] P. Goodyear and G. W. Hynd, "Attention-Deficit Disorder with $(\mathrm{ADD} / \mathrm{H})$ and without (ADD/WO) Hyperactivity: Behavioral and Neuropsychological Differentiation," Journal of Clinical Child Psychology, Vol. 21, No. 3, 1992, pp. 273-305. http://dx.doi.org/10.1207/s15374424jccp2103_9

[42] B. B. Lahey and C. L. Carlson, "Validity of the Diagnostic Category of Attention Deficit Disorder without Hyperactivity A Review of the Literature," Journal of Learning Disabilities, Vol. 24, No. 2, 1991, pp. 110-120. http://dx.doi.org/10.1177/002221949102400208
[43] P. Zimmermann and B. Fimm, "A Test Battery for Attentional Performance,” Applied Neuropsychology of Attention, Vol. 20, No. 2, 2002, pp. 110-151.

[44] B. A. Corbett, L. J. Constantine, R. Hendren, D. Rocke and S. Ozonoff, "Examining Executive Functioning in Children with Autism Spectrum Disorder, Attention Deficit Hyperactivity Disorder and Typical Development," Psychiatry Research, Vol. 166, No. 2, 2009, pp. 210-222. http://dx.doi.org/10.1016/j.psychres.2008.02.005

[45] R. Martinussen, J. Hayden, S. Hogg-Johnson and R. Tannock, "A Meta-Analysis of Working Memory Impairments in Children with Attention-Deficit/Hyperactivity Disorder," Journal of the American Academy of Child \& Adolescent Psychiatry, Vol. 44, No. 4, 2005, pp. 377-384. http://dx.doi.org/10.1097/01.chi.0000153228.72591.73

[46] H. M. Geurts, S. Verté, J. Oosterlaan, H. Roeyers and J. A. Sergean, "How Specific Are Executive Functioning Deficits in Attention Deficit Hyperactivity Disorder and Autism?” Journal of Child Psychology and Psychiatry, Vol. 45, No. 4, 2004, pp. 836-854.

http://dx.doi.org/10.1111/j.1469-7610.2004.00276.x

[47] H. M. Geurts, S. Verté, J. Oosterlaan, H. Roeyers and J. A. Sergean, "ADHD Subtypes: Do They Differ in Their Executive Functioning Profile?” Archives of Clinical Neuropsychology, Vol. 20, No. 4, 2005, pp. 457-477. http://dx.doi.org/10.1016/j.acn.2004.11.001

[48] B. F. Pennington, D. Groisser and M. C. Welsh, “Contrasting Cognitive Deficits in Attention Deficit Hyperactivity Disorder versus Reading Disability,” Developmental Psychology, Vol. 29, No. 3, 1993, pp. 511-523. http://dx.doi.org/10.1037/0012-1649.29.3.511

[49] L. L. Weyandt and W. G. Willis, "Executive Functions in School-Aged Children: Potential Efficacy of Tasks in Discriminating Clinical Groups,” Developmental Neuropsychology, Vol. 10, No. 1, 1994, pp. 27-38. http://dx.doi.org/10.1080/87565649409540564

[50] A. H. Amaral and M. M. Guerreiro, “Attention Deficit Hyperactivity Disorder: Proposal of Neuropsychological Assessment," Arquivos de Neuro-Psiquiatria, Vol. 59, No. 4, 2001, pp. 884-888.

[51] W. V. Reeve and S. L. Schandler, "Frontal Lobe Functioning in Adolescents with Attention Deficit Hyperactivity Disorder," Adolescence, Vol. 36, No. 144, 2001, pp. 749-765.

[52] M. Semrud-Clikeman, R. J. Steingard, P. Filipek, J. Biederman, K. Bekken and P. F. Renshaw, "Using MRI to Examine Brain-Behavior Relationships in Males with Attention Deficit Disorder with Hyperactivity," Journal of the American Academy of Child \& Adolescent Psychiatry, Vol. 39, No. 4, 2000, pp. 477-484. http://dx.doi.org/10.1097/00004583-200004000-00017

[53] G. Tripp, J. Ryan and K. Peace, "Neuropsychological Functioning in Children with DSM-IV Combined Type Attention Deficit Hyperactivity Disorder," Australian and New Zealand Journal of Psychiatry, Vol. 36, No. 6, 2002, pp. 771-779. http://dx.doi.org/10.1046/j.1440-1614.2002.01093.x

[54] S. Houghton, G. Douglas, J. West, et al., "Differential Patterns of Executive Function in Children with Attention- 
Deficit Hyperactivity Disorder According to gender and Subtype,” Journal of Child Neurology, Vol. 14, No. 12, 1999, pp. 801-805. http://dx.doi.org/10.1177/088307389901401206

[55] R. Van Mourik, J. Oosterlaan and J. A. Sergeant, "The Stroop Revisited: A Meta Analysis of Interference Control in AD/HD," Journal of Child Psychology and Psychiatry, Vol. 46, No. 2, 2005, pp. 150-165. http://dx.doi.org/10.1111/j.1469-7610.2004.00345.x

[56] M. M. Lansbergen, J. L. Kenemans and H. van Engeland, "Stroop Interference and Attention-Deficit/Hyperactivity Disorder: A Review and Meta-Analysis," Neuropsychology, Vol. 21, No. 2, 2007, pp. 251-262. http://dx.doi.org/10.1037/0894-4105.21.2.251

[57] J. R. Stroop, "Studies of Interference in Serial Verbal Reactions,” Journal of Experimental Psychology, Vol. 18, No. 6, 1935, pp. 643-662. http://dx.doi.org/10.1037/h0054651

[58] C. A. Riccio, S. Homack, K. P. Jarratt and M. E. Wolfe, "Differences in Academic and Executive Function Domains among Children with ADHD Predominantly Inat- tentive and Combined Types," Archives of Clinical Neuropsychology, Vol. 21, No. 7, 2006, pp. 657-667. http://dx.doi.org/10.1016/j.acn.2006.05.010

[59] P. G. Aaron, R. M. Joshi and J. Phipps, “A Cognitive Tool to Diagnose Predominantly Inattentive ADHD Behavior," Journal of Attention Disorders, Vol. 7, No. 3, 2004, pp. 125-135. http://dx.doi.org/10.1177/108705470400700301

[60] K. D. Gadow, J. Sprafkin and E. E. Nolan, "DSM-IV Symptoms in Community and Clinic Preschool Children,” Journal of the American Academy of Child \& Adolescent Psychiatry, Vol. 40, No. 12, 2001, pp. 1383-1392. http://dx.doi.org/10.1097/00004583-200112000-00008

[61] B. W. Graetz, M. G. Sawyer, P. L. Hazell, F. Arney and P. Baghurst, "Validity of DSM-IV ADHD Subtypes in a Nationally Representative Sample of Australian Children and Adolescents," Journal of the American Academy of Child \& Adolescent Psychiatry, Vol. 40, No. 12, 2001, pp. 1410-1417. http://dx.doi.org/10.1097/00004583-200112000-00011 